\title{
Journal of

\section{Optical and structural characterization of cerium doped LYSO sol-gel polycrystal films: potential application as scintillator panel for X-ray imaging $\dagger$}

\author{
P. C. Ricci, ${ }^{* a}$ C. M. Carbonaro,${ }^{a b}$ A. Casu, ${ }^{a}$ C. Cannas,,${ }^{c}$ R. Corpino, ${ }^{a b}$ L. Stagi ${ }^{a}$ and A. Anedda ${ }^{a b}$ \\ Received 31st January 2011, Accepted 22nd March 2011 \\ DOI: $10.1039 / \mathrm{c} 1 \mathrm{jm} 10492 \mathrm{f}$
}

\begin{abstract}
The optical and structural properties of sol-gel prepared polycrystalline films of Cerium doped Lutetium and Yttrium oxyorthosilicates (Ce:LYSO) are investigated by means of optical spectroscopy, XRD diffraction and Raman spectroscopy. The sol-gel samples are compared to commercially available Ce-doped LYSO single crystals, and a detailed study of the emission properties from the different cerium sites is performed. The main result is that the polycrystalline films do show very good radioluminescence properties for possible applications as X-ray scintillating panels. The structural characterization indicates that the sol-gel polycrystals have similar phase composition to commercial monocrystals; concerning the excitation and emission properties, the same radiative recombination channels are observed in the polycrystals and commercial monocrystals with a slightly faster decay time in the first case. In addition, low temperature measurements $(8 \mathrm{~K})$ indicate the presence of a nonradiative cross-relaxation mechanism among cerium ions in the sol-gel grown samples in the two possible crystallographic sites. The results indicate the possibility to develop sol-gel synthesized polycrystalline thin films as scintillating materials in the X-ray energy range.
\end{abstract}

\section{Introduction}

The family of Rare Earth ( $\mathrm{Re})$ doped crystal oxides is largely exploited in photonics as an active medium for solid state lasers and for the detection of high energy radiation. Within the family, Re oxyorthosilicates, $\mathrm{Re}_{2} \mathrm{SiO}_{5}$, and in particular cerium doped lutetium crystals $\left(\mathrm{Lu}_{2} \mathrm{SiO}_{5}, \mathrm{LSO}\right)$, yttrium crystals $\left(\mathrm{Y}_{2} \mathrm{SiO}_{5}\right.$, YSO) and their solid solutions $\left(\mathrm{Lu}_{2-x} \mathrm{Y}_{x} \mathrm{SiO}_{5}, \mathrm{LYSO}\right)$, gained a far-reaching role because of the application as scintillators for ionizing radiations. ${ }^{1-3}$ The leading characteristics of these materials are the high stopping power for high energy photons (gamma at $511 \mathrm{KeV}$ ), successfully employed in the positron emission tomography (PET), and the scintillation time of about $40 \mathrm{~ns}$, much lower than other widely used conventional scintillators (i.e. BGO $300 \mathrm{~ns}$, CsI $900 \mathrm{~ns}$ ). ${ }^{4}$ Despite the numerous and successful applications, ${ }^{5,6}$ there are a few unsolved drawbacks boosting the research to improve the material features and to develop new devices and technologies. The main one is related to the growing process: these materials are typically grown as large

${ }^{a}$ Dipartimento di Fisica, Università di Cagliari, s.p. n 8 Km 0.700, 09042 Monserrato, (Cagliari), Italy

${ }^{b}$ Centro Grandi Strumenti Università di Cagliari, s.p. $n 8$ Km 0.700, 09042 Monserrato, (Cagliari), Italy

'Dipartimento di Scienze Chimiche, Università di Cagliari, Cittadella Universitaria, 09042 Monserrato, (Cagliari), Italy

$\uparrow$ Electronic supplementary information (ESI) available. See DOI: $10.1039 / \mathrm{c} 1 \mathrm{jm} 10492 \mathrm{f}$ single crystals by the Czochralski technique reaching very high temperature of crystallisation (larger than $2000{ }^{\circ} \mathrm{C}$ ). Thus the development of more convenient approaches to produce this class of materials is a research and technology challenge towards less expensive scintillators. Recently the production of nanopowders by means of solution combustion synthesis proved to be efficient in reducing the afterglow of scintillators and in increasing the concentration quenching of Ce dopant ions up to $1 \%$, further enhancing their optical performances. ${ }^{7,8}$ Among the possibilities, the sol-gel synthesis is one of the most attractive techniques, since it allows to obtain polycrystals at a considerably lower temperature than the Czochralski technique. By means of sol-gel synthesis the prepared polycrystals can easily be shaped as scintillating films, particularly valuable in fundamental spectroscopic studies and for high resolution imaging in X-ray scintillating panels. In the mammography field, for example, the delivered radiation dose is of the order of a few mGy and the estimated X-ray penetration depth in our samples, by assuming a reduction of the incident radiation of about 2 orders of magnitude, is of the order of $600 \mu \mathrm{m}$. Taking carefully into account the scattered radiation, one can hypothesize that a $1 \mathrm{~mm}$ thick sample of about $50 \mathrm{mg} \mathrm{cm}^{-2}$ weight could be exploited to produce a sol-gel based detecting device. In a recent paper we have reported preliminary results on the emission properties of sol-gel derived cerium doped yttrium and lutetium oxyorthosilicate showing that the investigated nanopowders could be successfully exploited in the soft X-ray detection. ${ }^{9}$ 
However, despite the large interest in single crystal scintillators, there are only few and recent papers concerning the production and characterization of sol-gel derived scintillators. ${ }^{6,9,10,11}$ Therefore, it is important to compare the luminescence properties of sol-gel synthesized nanopowders of oxyorthosilicates to single crystals from both the basic and the applied points of view.

In this work we present the optical properties of sol-gel derived cerium doped yttrium and lutetium $\left(\mathrm{Ce}: \mathrm{Lu}_{1.2} \mathrm{Y}_{0.8} \mathrm{SiO}_{5}\right)$ oxyorthosilicate investigated by means of low $(8 \mathrm{~K})$ and room temperature VUV and visible spectroscopy. Steady state and time resolved luminescence (PL), Raman spectra and X-ray diffraction patterns are also presented. The results are compared and analysed with respect to the optical properties of commercially available single crystals. Finally we show a direct application of sol-gel based Ce:LYSO thin film as a panel for soft $\mathrm{X}$-ray radiography.

\section{Experimental}

Single crystal samples of cerium doped $\mathrm{Lu}_{1.2} \mathrm{Y}_{0.8} \mathrm{SiO}_{5}$ for commercial applications were grown by the Czochralski method by SAES Opto Materials. Pure oxides of $\mathrm{Lu}_{2} \mathrm{O}_{3}$ (99.999\%), $\mathrm{Y}_{2} \mathrm{O}_{3}(99.999 \%)$ and $\mathrm{SiO}_{2}(99.999 \%)$ and $\mathrm{CeO}_{2}$ were pre-sintered under a pressure of $140 \mathrm{MPa}$ and processed at $1400{ }^{\circ} \mathrm{C}$ for 24 hours; sintered tablets were consequently melt up to $2150^{\circ} \mathrm{C}$. The obtained undoped boule was used as a seed for pulling a Cedoped melt. The samples were cut along the [100] direction and lapped to optical finish. The sol-gel synthesized samples were prepared starting from the same rare earth powder oxides of the single crystal samples. The powders were dissolved in a mixture of $\mathrm{H}_{2} \mathrm{O}$ and $\mathrm{HNO}_{3}$. The solution, kept under stirring, was added to equal volumes of ethyl alcohol and TEOS. A gel was precipitated by adding $\mathrm{NH}_{4} \mathrm{OH}$, and dried for 3 days at $70{ }^{\circ} \mathrm{C}$. The dried gel was treated for $2 \mathrm{~h}$ at $200{ }^{\circ} \mathrm{C}, 300{ }^{\circ} \mathrm{C}, 500{ }^{\circ} \mathrm{C}$ and $700{ }^{\circ} \mathrm{C}$, for $100 \mathrm{~h}$ at $1300^{\circ} \mathrm{C}$ and finally for $4 \mathrm{~h}$ at $1400{ }^{\circ} \mathrm{C}$. The synthesized powder was dispersed in a collodion solution of cellulose nitrate ( $2 \%$ in amyl acetate, Fluka Analytical), kept under stirring and then deposited on an optically transparent polymeric substrate (Makrolon, Bayer) by the solvent-assisted drop-casting technique and a $3 \times 2 \mathrm{~cm}^{2}$ panel with a thickness of about $800 \mu \mathrm{m}$ was obtained.

Samples with cerium concentration of about $0.1 \%$ in weight were analysed. In the following we will refer to the Czochralski grown single crystal samples as CZ, and to sol-gel polycrystalline samples as SG.

Photoluminescence (PL) measurements were carried out at the SUPERLUMI experimental station on the I beamline of the HASYLAB synchrotron laboratories at DESY (Hamburg), the excitation light being provided by the pulsed synchrotron radiation. The PL signal was dispersed by a Spectra-Pro $300 \mathrm{i}$ monochromator and detected in the $250-750 \mathrm{~nm}$ range with a LN-CCD (Princeton Instruments). PL spectra were recorded at room temperature under multi-bunch operation and detected with an integral time window of 192 ns correlated to the SR pulses. Time resolved PL measurements were performed using an optical parametric oscillator with a frequency doubler device, excited by the third harmonic $(355 \mathrm{~nm})$ of a pulsed Nd-YAG laser (Quanta-Ray Pro 730). The excitation pulse width at half maximum was $8 \mathrm{~ns}$ with $10 \mathrm{~Hz}$ of repetition rate, its power density ranged between 25 and $75 \mathrm{~mW} \mathrm{~cm}^{-2}$ and the spectral bandwidth was $<0.3 \mathrm{~cm}^{-1}$. The PL signal was dispersed by a spectrograph (ARC-SpectraPro 300i) with a spectral bandpass $<2.5 \mathrm{~nm}$ and detected by a gateable intensified CCD (PI MAX Princeton Inst.). Spectra were corrected for the optical transfer function.

Excitation of PL (PLE) measurements was carried out in the $150-400 \mathrm{~nm}$ range by exciting the sample with the light provided by a $\mathrm{MgF}_{2}$-sealed deuterium lamp (Hamamatsu mod. L1835) coupled with a 0.3 metre vacuum ultraviolet scanning monochromator (McPherson mod. 218) under computer control (spectral bandwidth $1 \mathrm{~nm}$ ). The excitation light was filtered with a WG-295 glass filter in the $300-400 \mathrm{~nm}$ range. The PL signal was passed through a selected interferential filter (centre wavelength 400 and $460 \mathrm{~nm}$, bandwidth $10 \mathrm{~nm}$ ) and was gathered with a photomultiplier (EMI 9558 QB). Spectra were corrected for the optical transfer function.

Raman scattering measurements were carried out in back scattering geometry along the principal crystal directions by using the $514.5 \mathrm{~nm}$ line of an argon-ion laser. Raman spectra were performed in air at room temperature with a triple spectrometer Jobin-Yvon Dilor integrated system with a spectral resolution of about $1 \mathrm{~cm}^{-1}$. Spectra have been recorded in the Stokes region by a 1200 grooves per $\mathrm{mm}$ grating monochromator and a CCD detector system. X-Ray powder diffraction patterns were performed by using a $\vartheta-\vartheta$ diffractometer (Seifert X3000) with Bragg-Brentano geometry and $\mathrm{Cu} \mathrm{K} \alpha$ radiation. Before the measurements, the samples were grinded with agate mortar and the obtained fine powders were dispersed in ethanol, sonicated, deposited drop by drop on a silicon zero background sample holder and dried in air. ${ }^{12}$

\section{Results and discussion}

The crystal structure of a solid solution of $\mathrm{Re}_{2} \mathrm{SiO}_{5}$ oxyorthosilicates depends on the ionic radius of the trivalent ions $\mathrm{Re}^{3+}$, the growing temperature or the pressure. ${ }^{13-15}$ The two possible structures, belonging to the $P 21 / c$ and $C 2 / c$ space groups respectively, have a monoclinic symmetry and differ in the oxygen coordination of rare earth cations. In the first case the structure of isolated tetrahedra $\left(\mathrm{SiO}_{4}\right)$ presents an oxygen site not silicon-bonded and two crystallographically independent $\mathrm{Re}^{3+}$ sites. The extra oxygen atoms are located at the centre of tetrahedra arranged in a two dimensional network parallel to the (100) plane. ${ }^{13}$ The $C 2 / c$ crystal structure is typically achieved with $\mathrm{Re}^{3+}$ elements with smaller ionic radius and is obtained by four $\mathrm{Re}_{2} \mathrm{SiO}_{5}$ formula units in a B-centred monoclinic unit cell. Similar to the $P 2 / 1 c$ structure, it presents two independent crystallographic sites for the principal rare earth, coordinated with 6 and 7 oxygen ions respectively.

In this framework, $\mathrm{Lu}_{2} \mathrm{SiO}_{5}$ crystallizes in the $C 2 / c$ structure while the structure of pure yttrium oxyorthosilicates depends on the growth conditions: it crystallizes in the $P 21 / c$ structure when annealed at temperatures lower than $1190{ }^{\circ} \mathrm{C}$, otherwise in the $C 2 / c$ space group. ${ }^{16,17}$ The structure of LYSO monocrystal, grown by the Czochralski technique, was proven to belong to the monoclinic $C 2 / c$ symmetry group. ${ }^{14}$ The irreducible point representations of this symmetry group allow 96 Raman active vibrational modes (on a total of 192) with $48 \mathrm{Ag}$ and $48 \mathrm{Bg}$ 


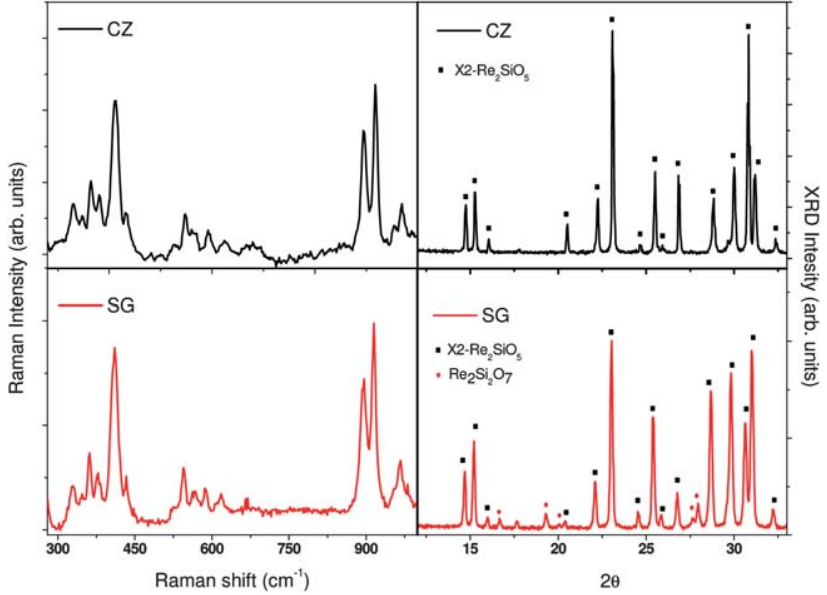

Fig. 1 Unpolarized Raman spectra (left) and X-ray diffraction patterns (right) of the Czochralski (CZ) and sol-gel (SG) LYSO samples.

Table 1 Main vibrational modes detected in LYSO samples (most intense modes are labeled with "*”)

\begin{tabular}{ll}
\hline Raman modes $/ \mathrm{cm}^{-1}$ \\
\hline$A_{\mathrm{g}}$ & $338,376,402^{*}, 426,487,541^{*}, 617,889^{*}, 911^{*}, 962$ \\
$B_{\mathrm{g}}$ & $264,321,356^{*}, 408^{*}, 435,514,560,585^{*}, 949$
\end{tabular}

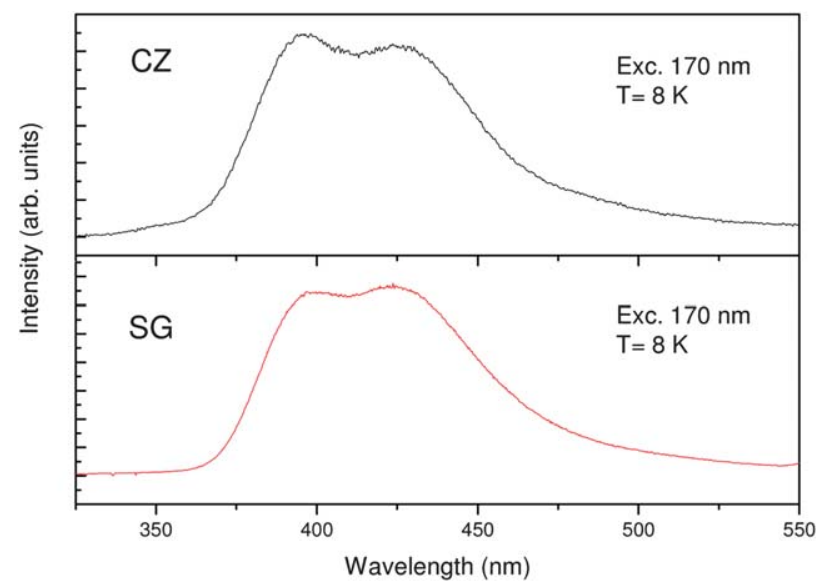

Fig. 2 PL spectra of Czochralski (CZ) and sol-gel (SG) LYSO samples at low temperature $(8 \mathrm{~K})$ excited at $170 \mathrm{~nm}$.

modes. ${ }^{15,20}$ Fig. 1 (left) presents the unpolarized Raman spectra of LYSO CZ (upper panel) and SG (lower panel) samples. Both the spectra display different bands in the range from 60 to $1000 \mathrm{~cm}^{-1}$, where some peaks are not resolved and overlapped. The two spectra are very similar and all the frequency peaks of the vibrational modes of the sol-gel samples do coincide with those of the $\mathrm{CZ}$ ones, allowing to assign the sol-gel samples to the $C 2 / c$ symmetry group, as the $\mathrm{CZ}$ samples (Table 1). The assignment is further confirmed by X-ray diffraction patterns (Fig. 1 - right) showing that the sol-gel powders have a main crystalline phase composition similar to the monocrystals. Beside the main reflections of the $\mathrm{Re}_{2} \mathrm{SiO}_{5} \mathrm{C} 2 / c$ phase, the SG samples show a few low intensity peaks indicating the presence of a small amount of a secondary phase attributable to the presence of lutetium pyrosilicates $\left(\mathrm{Re}_{2} \mathrm{Si}_{2} \mathrm{O}_{7}\right) \cdot{ }^{18}$ No other phases, like $\mathrm{Lu}_{2} \mathrm{O}_{3}$, $\mathrm{Y}_{2} \mathrm{O}_{3}, \mathrm{Y} 4.67\left(\mathrm{SiO}_{4}\right)_{3} \mathrm{O}$ and $\mathrm{Ce}_{2} \mathrm{O}_{3}$, are detectable.

The optical properties of the two classes of samples were analyzed by comparing the PL spectra related to the main cerium emission in the $400-500 \mathrm{~nm}$ spectral range (Fig. 2). ${ }^{2}$ The Cerelated overall PL spectra, excited at $170 \mathrm{~nm}$ at low temperature $(8 \mathrm{~K})$, show two clear maxima with the intensity ratio between them reversed in the case of SG and CZ samples. It is well known that $\mathrm{Ce}$ ions can substitute the bulk rare earth elements ( $\mathrm{Lu}$ and $\mathrm{Y}$ in this case) in two sites: the so-called Re1 site has seven nearest neighbour $\mathrm{O}$ atoms and the PL band is peaked at $394 \mathrm{~nm}$ while the Re2 site is bound to only six oxygen atoms and the PL peak is centred at $460 \mathrm{~nm} .{ }^{19}$ The slight difference in the emission spectra of the SG sample excited with high energy light could be attributed to a different contribution of the Re1 and Re 2 emissions with respect to the monocrystalline sample. The presence of two Ce-related emitting centres is confirmed by site selective PLE spectra of SG sample (Fig. 3), showing two maxima at 260 and $295 \mathrm{~nm}$ for the emission from the Ce1 site and at about $320 \mathrm{~nm}$ for the emission at lower energy ( $\mathrm{Ce} 2$ site). The reported results are in good agreement with previous results obtained for monocrystal samples. ${ }^{2,6,21,22}$

In order to better understand the different optical properties of the two classes of samples, low temperature (8 K) PL measurements were performed (Fig. 4). The contribution from each site was partially isolated by site selective excitation at $295 \mathrm{~nm}$ for the $\mathrm{Ce} 1$ site and at $320 \mathrm{~nm}$ for the $\mathrm{Ce} 2$ site. The spectra arising from the $295 \mathrm{~nm}$ excitation for the two classes of samples are very similar to each other (Fig. 4A) while considerable differences in the two emission spectra excited at $320 \mathrm{~nm}$ are observed (Fig. 4B). In these spectra the emission from the $\mathrm{CZ}$ samples can be assigned solely to the recombination from the $\mathrm{Ce} 2$ site, because of a negligible spectral overlapping with the PL from Ce1, while the emission spectrum of the SG sample, excited at $320 \mathrm{~nm}$, shows a large contribution from other recombination sites. In order to highlight all the emission components from the SG sample, the following spectral subtraction procedure was applied: the PL spectrum excited at $295 \mathrm{~nm}$, previously assigned to the selective recombination at the Cel site (Fig. 4A), was scaled to the PL intensity at $394 \mathrm{~nm}$ in the spectrum recorded

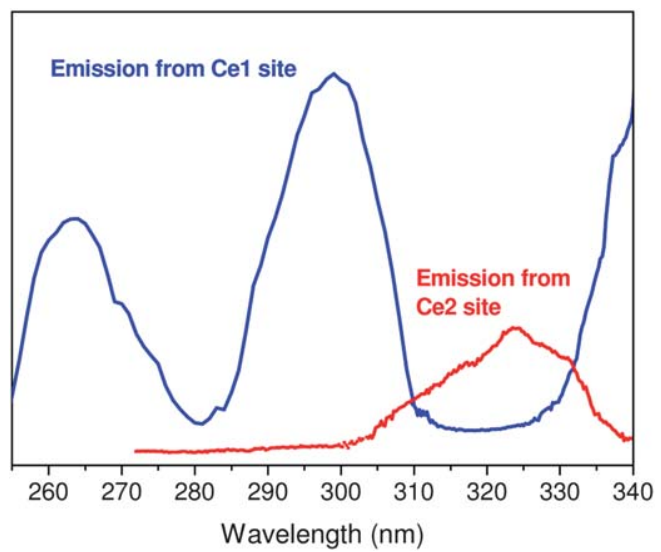

Fig. 3 PLE spectra of sol-gel samples recorded by monitoring at $400 \mathrm{~nm}$ (Ce1 site) and $460 \mathrm{~nm}$ (Ce2 site). 


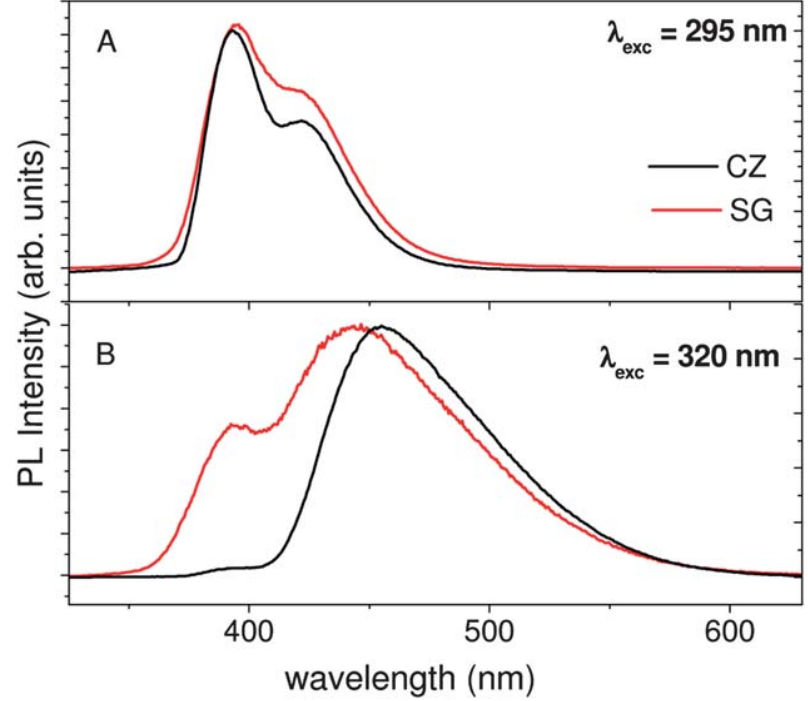

Fig. 4 PL spectra of Czochralski (CZ) and sol-gel (SG) LYSO samples. Excitation wavelengths are $295 \mathrm{~nm}$ (A) and $320 \mathrm{~nm}$ (B).

under $320 \mathrm{~nm}$ excitation (Fig. 5A). The scaled Ce1 PL spectrum was successively subtracted from the PL spectrum excited at $320 \mathrm{~nm}$. The result of the spectral subtraction (Fig. 5B) is a spectrum that strictly resembles the PL spectrum of the CZ sample excited at $320 \mathrm{~nm}$, the one previously assigned to the selective recombination at $\mathrm{Ce} 2$ site (Fig. 4B). The applied procedure allows recognition of the presence of only two radiative recombination components arising from the two inequivalent cerium sites, $\mathrm{Ce} 1$ and $\mathrm{Ce} 2$. In order to achieve a deeper insight in the above discussed assignment a Gaussian fit analysis of the collected spectra was carried out. Two Gaussian bands for each site (Fig. 6) were used as basis functions, since each contribution to the PL at the cerium sites arises from the recombinations from the $5 \mathrm{~d}$ levels to the fundamental doubly split 4f levels ${ }^{2} \mathrm{~F}_{5 / 2}$ and ${ }^{2} \mathrm{~F}_{7 / 2}$ (see Table 2). Moreover the analytical reconstruction allows estimation and comparison of the spin-orbit energy split of the cerium F-levels in lutetium yttrium oxyorthosilicates by the evaluation of the energy difference in the PL peak of the Gaussian components: the spin-orbit

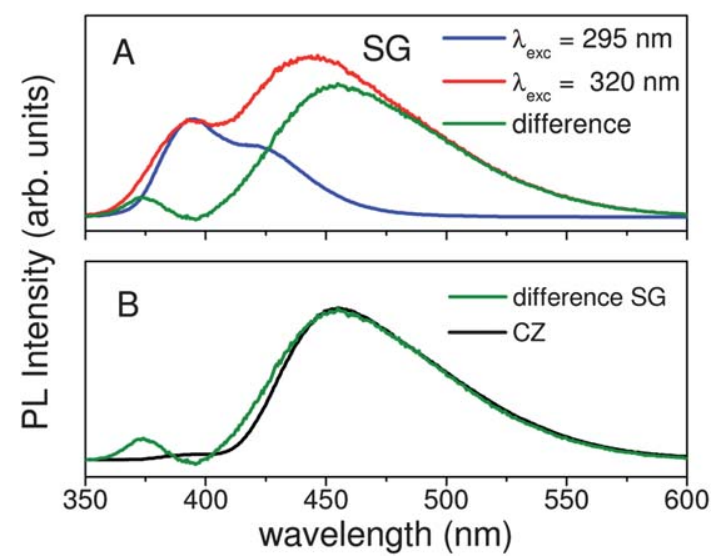

Fig. 5 (A) Normalized PL spectra of sol-gel (SG) LYSO samples and result of their spectral subtraction (see text for details). (B) Comparison between CZ PL spectrum and difference PL spectrum.

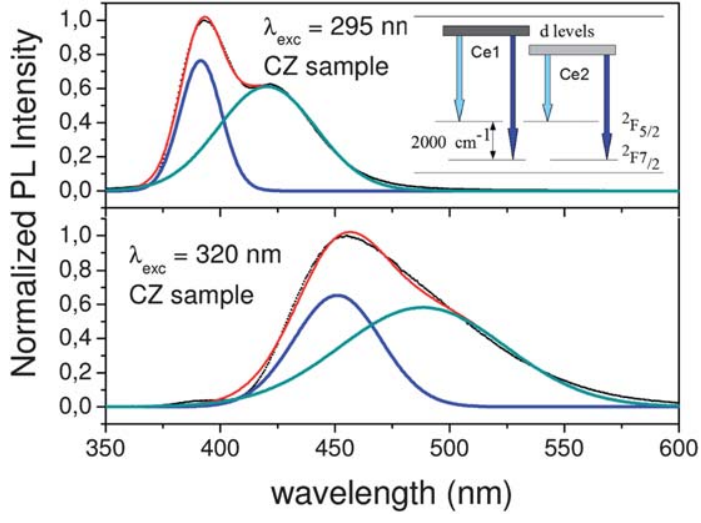

Fig. 6 Curve-fitting analysis (solid red line) of the PL spectra (black triangles). Two Gaussian curves (blue and green solid lines) for each site were used as basis functions and the relative scheme of energy levels is reported.

energy split was estimated to be of about $2000 \mathrm{~cm}^{-1}$ in both the classes of samples. The high resemblance of the site selected photoluminescence spectra of the two classes of samples is a further indication of the high similarity of the crystal structure and of the similar optical characteristics of the Ce dopant inside LYSO matrix.

To complete the optical characterization of the samples and to understand the dynamics of the recombination at the cerium sites, site selective time resolved measurements were performed by exciting at $295 \mathrm{~nm}$ and $320 \mathrm{~nm}$ at $8 \mathrm{~K}$. A single exponential decay time was observed for all the recorded emissions, as reported in Table 3. The analysis shows that the decay time of the Ce1 site PL was 33 ns for both poly- and mono-crystal samples, while the lifetime constants for $\mathrm{Ce} 2$ site were $46 \mathrm{~ns}$ and $38 \mathrm{~ns}$, for the $\mathrm{CZ}$ and SG sample respectively (Fig. 7). Similar results were obtained for room temperature time resolved PL measurements. The faster kinetics observed in the polycrystals can be interpreted by taking into account the differences highlighted in the PL spectra by site selective excitation at $320 \mathrm{~nm}$ previously discussed. Indeed while the excitation at $320 \mathrm{~nm}$ of single crystals induces PL emission mainly from the $\mathrm{Ce} 2$ sites, with almost no signal from the Cel sites, the same excitation in polycrystals induces a remarkable emission from the $\mathrm{Ce} 1$ site, accompanied by a decrease in PL lifetime of the $\mathrm{Ce} 2$ sites. The correlation of the $\mathrm{Ce} 1$ and $\mathrm{Ce} 2$ sites observed in the polycrystalline samples is indicative of a non-radiative cross-relaxation mechanism among cerium ions belonging to different crystalline sites.

Finally, we show a direct application of a sol-gel based Ce: LYSO thin film as a panel for soft X-ray radiography. Fig. 8 shows two images from the X-ray irradiated panel, through

Table 2 Spectral features (peak position and Full Width at Half Maximum, FWHM) of the two fitting Gaussian bands of PL spectra (Fig. 6) for Czochralski and sol-gel (between brackets) samples under 295 and $320 \mathrm{~nm}$ excitations

\begin{tabular}{llcll}
\hline & $\begin{array}{l}\lambda_{\mathrm{exc}}=295 / \\
\mathrm{nm}\end{array}$ & & $\begin{array}{l}\lambda_{\mathrm{exc}}=320 / \\
\mathrm{nm}\end{array}$ \\
\hline Peak/nm & $391(391)$ & $420(420)$ & $451(449)$ & $488(488)$ \\
FWHM/nm & $18(19)$ & $42(46)$ & $37(42)$ & $71(74)$ \\
\hline
\end{tabular}


Table 3 Photoluminescence lifetimes of Czochralski and sol-gel (between brackets) samples with different excitation wavelengths

\begin{tabular}{llll}
\hline & & $\tau / \mathrm{ns}$, & $\tau / \mathrm{ns}$, \\
Excitation/nm & Emission/nm & $8 \mathrm{~K}$ & $290 \mathrm{~K}$ \\
\hline 295 & $393(393)$ & $33(33)$ & $30(30)$ \\
320 & $460(460)$ & $46(38)$ & $36(32)$ \\
\hline
\end{tabular}

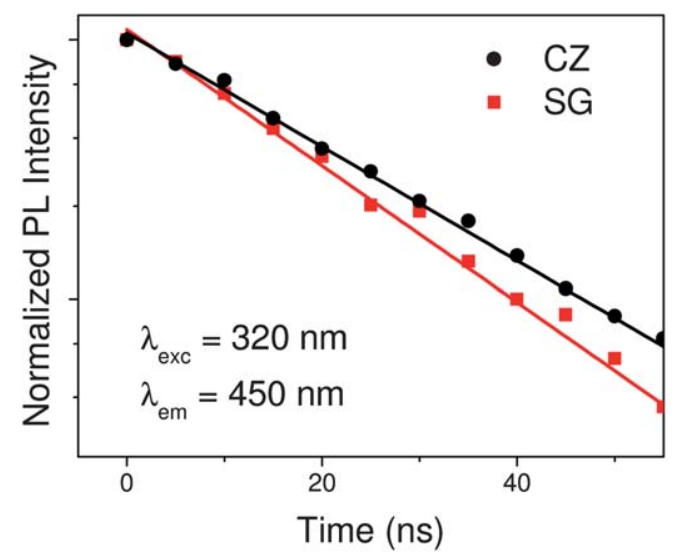

Fig. 7 Time-resolved photoluminescence measurements and single exponential fit (solid line) of Czochralski (CZ) and sol-gel (SG) samples (excitation at $320 \mathrm{~nm}$, PL detected at $450 \mathrm{~nm}$ ).

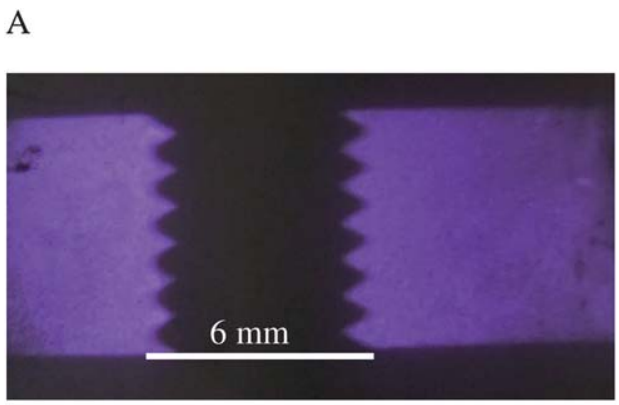

B
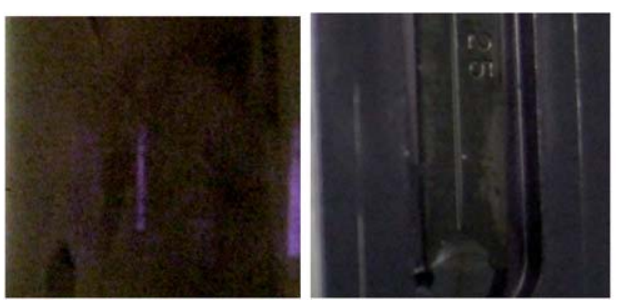

Fig. 8 Images recorded by irradiating a sol-gel thick film with soft Xray: a screw (A) and a $25 \mathrm{~mm}$ slit (B), the right panel reports the slit for reference.

a screw (A) and a $25 \mu \mathrm{m}$ slit (B, right panel reports the slit for reference), respectively. The samples were irradiated with a standard X-ray source, operating at $25 \mathrm{kV}$ and $20 \mathrm{~mA}(\mathrm{Cu}$ anode) and $1 / 4 \mathrm{~s}$ accumulation time was used to impress the radiography on a standard CCD camera (Fuji S200 EXR). To confirm the proposed application we also collected the same images on a tick panel of $\mathrm{CZ}$ grown sample (see ESI $\dagger$ ) obtaining comparable results. The good signal to noise ratio and the high spatial resolution of the pictures allow us to propose sol-gel based Ce:LYSO samples as good candidates of a new generation material for digital radiography, for which the realization of the single crystal panel and their cost are not comparable to the ones of sol-gel synthesized samples.

\section{Conclusions}

The optical and structural properties of sol-gel prepared polycrystalline Ce-doped LYSO samples were characterized by means of photoluminescence spectroscopy, X-ray diffraction, and Raman measurements. The results were compared to the structure and optical features of commercially available monocrystals grown by the Czochralski technique. The structural analysis reveals the same symmetry group for the two classes of crystals. By comparing the collected photoluminescence data it was shown that the sol-gel polycrystalline samples exhibit a nonradiative cross-relaxation mechanism among cerium ions assignable to the quenching effect in the $\mathrm{Ce} 2$ site. These findings suggest that, from a spectroscopic point of view, sol-gel prepared LYSO can be an alternative to Czochralski grown samples as useful materials in the detection of ionizing radiations and for scintillating devices in the soft X-ray energy range.

\section{Acknowledgements}

The research leading to these results has received funding from the European Community's Seventh Framwork Programme (FP7/2007-2013) under grant agreement no. 226716. P. C. Ricci is grateful for the economic support from the RAS (Regione Autonoma della Sardegna) and European community through the Grants in aid for Young Researcher, L.R. no. 7, 2007, no. CRP 2_539.

\section{References}

1 C. L. Melcher, M. Schmand, M. Eriksson, L. Eriksson, M. Casey, R. Nutt, J. Lefaucheur and B. Chai, IEEE Trans. Nucl. Sci., 2000, 47, 965 .

2 A. J. Wojtowicz, W. Drozdowski, D. Wisniewski, J. Lefaucheur, Z. Galazka, Z. Gou, T. Lukasiewicz and J. Kisielewski, Opt. Mater., 2006, 28, 85.

3 P. Szupryczynski, C. L. Melcher, M. A. Spurrier, M. P. Maskarinec, A. A. Carey, A. J. Wojtowicz, W. Drozdowski, D. Wisniewski and R. Nutt, IEEE Trans. Nucl. Sci., 2004, 51, 1103.

4 J. K. Lee, R. E. Muenchausen, J. S. Lee, Q. X. Jia, M. Nastasi, J. A. Valdez, B. L. Bennett and D. W. Cooke, Appl. Phys. Lett., 2006, 89, 101905.

5 B. Hautefille, K. Lebbou, C. Dujardin, J. M. Fourmigue, L. L. Grosvalet, O. Tillement and C. Pedrini, J. Cryst. Growth, 2006, 289, 172.

6 L. S. Qin, Y. T. Wu, H. S. Shi, W. X. Chai, K. Y. Shu, G. H. Ren and X. F. Chen, IEEE Trans. Nucl. Sci., 2009, 56, 2979.

7 E. G. Yukihara, L. G. Jacobsohn, M. W. Blair, B. L. Bennett, S. C. Tornga and R. E. Muenchausen, J. Lumin., 2010, 130, 2309.

8 R. E. Muenchausen, E. A. McKigney, L. G. Jacobsohn, M. W. Blair, B. L. Bennett and D. W. Cooke, IEEE Trans. Nucl. Sci., 2008, 55, 1532.

9 P. C. Ricci, C. M. Carbonaro, L. Stagi, A. Anedda, F. Ferrari, D. Capsoni, A. Magistris, J. Non-cryst. Solids, DOI: 10.1016/j. jnoncrysol.2010.11.106.

10 P. Yun, Y. Shi, D. Zhou and J. Xie, J. Rare Earths, 2009, 27, 801. 
11 H. Wu, M. H. Engelhard, J. Wang, D. R. Fisher and Y. Lin, J. Mater Chem., 2008, 18, 1779.

12 N. Masciocchi, International Workshop on Structural Determination from Powder Diffraction Data, Villigen (CH), June 18-22, 2008.

13 J. Felsche, Struct. Bonding, 1973, 13, 99.

14 D. Chiriu, N. Faedda, A. Geddo Lehmann, P. C. Ricci, A. Anedda, S. Desgreniers and E. Fortin, Phys. Rev. B: Condens. Matter Mater. Phys., 2007, B76, 054112.

15 P. C. Ricci, D. Chiriu, C. M. Carbonaro, S. Desgreniers, E. Fortin and A. Anedda, J. Raman Spectrosc., 2008, 39, 1268.

16 C. Cannas, A. Musinu, G. Piccaluga, C. Deidda, F. Serra, M. Bazzoni and S. Enzo, J. Solid State Chem., 2005, 178, 1526.
17 C. Cannas, M. Mainas, A. Musinu, G. Piccaluga, S. Enzo, M. Bazzoni, A. Speghini and M. Bettinelli, Opt. Mater., 2007, 29, 585. 18 F. Bretheau-Raynal, N. Tercier, B. Blanzat and M. Drifford, Mater. Res. Bull., 1980, 15, 639.

19 W. Drozdowski, A. J.Wojtowicz, D. Wisniewski, P. Szupryczynski, S. Janus, J. L. Lefaucheur and Z. Gou, J. Alloys Compd., 2004, 380, 146. 20 A. Ellens, H. Andres, A. Meijerink and G. Blasse, Phys. Rev. B: Condens. Matter Mater. Phys., 1997, 55, 173.

21 P. C. Ricci, C. M. Carbonaro, D. Chiriu, R. Corpino, N. Faedda, M. Marceddu and A. Anedda, Mater. Sci. Eng., B, 2008, 146, 2.

22 D. W. Cooke, K. J. McClellan, B. L. Bennett, J. M. Roper, M. T. Whittaker and R. C. Sze, J. Appl. Phys., 2000, 88, 7360. 